\title{
Climate, Tectonics and Soil Production
}

\author{
ARJUN M. HEIMSATH \\ 'School or Earth and Space Exploration, ASU, Tempe, AZ \\ 85287
}

Soil production (SP) rates were first quantified using in situ produced cosmogenic nuclides (primarily $10-\mathrm{Be}$, but also 26-Al and 36-Cl) twenty-five years ago. Initial efforts focused on testing the hypothesis that soil production rates declined with increasing soil thickness, quantifying the soil production function. Subsequent and extensive work focuses on teasing apart the state factor matrix, especially on the variables of climate (i.e. precipitation, temperature and biota) and tectonics (i.e. slope, erosion or uplift rate, etc.). Oliver Chadwick's hand stirred the mix of many of these efforts, both directly and indirectly and this presentation pays tribute to his contributions by focusing primarily on the climatic and tectonic forcing of soil production.

The thickness of mobile regolith or "colluvial soil" is a fundamentally important characteristic of the critical zone that plays an essential role in hydrology, ecology, the action of bio-geochemical cycles, and erosion and transport processes. We use the term "colluvial soil", often shortened to simply "soil", rather than the equivalent "mobile regolith" because it is deeply entrenched in the geomorphic literature. In our usage soil production is the process of mechanical breakdown and entrainment of rock or regolith into the mobile, active colluvial soil - soil production thus is distinct from pedogenesis and governed by different processes. The thickness of such colluvial soil on hillslopes reflects a balance between the rate or erosion and the rate of soil production at the soil/weathered rock interface.

Here, we present data from a range of studies that specifically test the roles that climate and tectonics play in driving SP rates by keeping the other state factor variables as constant across field sites as possible. Results definitively show that SP rates scale directly with catchment averaged erosion rates in tectonically active settings. Samples from the more rapidly eroding landscapes define a SP function that is markedly steeper, with a significantly higher SP rate efficiency than the SP function defined by samples from the more slowly eroding landscapes. Results from tectonically quiescent landscapes with variable climates also show a difference between steep and gentle topography, but the dependence of SP on soil thickness is equivocal, suggesting the potential for equilibrium landscapes rather than the disequilibrium implied by SP functions quantified in tectonically active regions. 